\title{
S-wave attenuation structure beneath the northern Izu-Bonin arc
}

\author{
Tsutomu Takahashi", Koichiro Obana and Shuichi Kodaira
}

\begin{abstract}
To understand temperature structure or magma distribution in the crust and uppermost mantle, it is essential to know their attenuation structure. This study estimated the 3-D S-wave attenuation structure in the crust and uppermost mantle at the northern Izu-Bonin arc, taking into account the apparent attenuation due to multiple forward scattering. In the uppermost mantle, two areas of high seismic attenuation (high $Q^{-1}$ ) imaged beneath the volcanic front were mostly colocated with low-velocity anomalies. This coincidence suggests that these high- $Q^{-1}$ areas in low-velocity zones are the most likely candidates for high-temperature regions beneath volcanoes. The distribution of random inhomogeneities indicated the presence of three anomalies beneath the volcanic front: Two were in high$Q^{-1}$ areas but the third was in a moderate- $Q^{-1}$ area, indicating a low correlation between random inhomogeneities and $Q^{-1}$. All three anomalies of random inhomogeneities were rich in short-wavelength spectra. The most probable interpretation of such spectra is the presence of volcanic rock, which would be related to accumulated magma intrusion during episodes of volcanic activity. Therefore, the different distributions of $Q^{-1}$ and random inhomogeneities imply that the positions of hot regions in the uppermost mantle beneath this arc have changed temporally; therefore, they may provide important constraints on the evolutionary processes of arc crust and volcanoes.
\end{abstract}

Keywords: Seismic wave attenuation, Random inhomogeneity, Quaternary volcano

\section{Background}

The Izu-Bonin arc is an intraoceanic island arc on the Philippine Sea plate (Fig. 1). Seismic reflection and refraction surveys have been conducted extensively around this arc to elucidate its crustal structure and evolution processes. An important seismic structure in the Izu-Bonin arc is middle crust $\left(V_{\mathrm{p}}=6.0-6.8 \mathrm{~km} / \mathrm{s}\right)$, which was first found by a seismic survey across the volcanic front of the northern arc (Suyehiro et al. 1998). Middle crust is a representative feature of continental crust; therefore, this island arc is considered to be an ideal region for field studies of arc evolution and continental crust generation. Subsequent seismic surveys identified middle crust along many survey lines around this arc and showed that beneath the volcanic front the middle crust tends to be thick beneath basaltic volcanoes (e.g., Kodaira et al. 2007a, b). This clear correlation between volcanoes

*Correspondence: ttaka@jamstec.go.jp

Japan Agency for Marine-Earth Science and Technology, Showa-machi 3173-25, Kanazawa-ku, Yokohama 236-0001, Japan and crustal structure is a significant constraint on middle-crust generation and arc evolution processes (e.g., Tatsumi et al. 2008).

The uppermost mantle structure beneath this arc is an important boundary condition for evolutionary processes of the volcanoes and crustal structures. Obana et al. (2010) examined the crust and uppermost mantle structures beneath the arc by passive seismic observation with ocean bottom seismographs (OBSs). They focused on the northern part of the Izu-Bonin arc from Hachijo-jima to Tori-shima (Fig. 1), where the middle crust beneath the basaltic volcanoes tends to be thicker compared with the southern part (Kodaira et al. 2007b). Their travel time tomography with seismic data of the OBSs and onshore stations revealed several low-velocity anomalies of P- and $\mathrm{S}$-waves in the mantle wedge beneath the volcanic front. These low-velocity anomalies are presumably related to the magma distribution, but the horizontal extent of these low-velocity zones was much larger than that of the thick middle crust; therefore, the relationship between these velocity anomalies and the volcano distribution was 


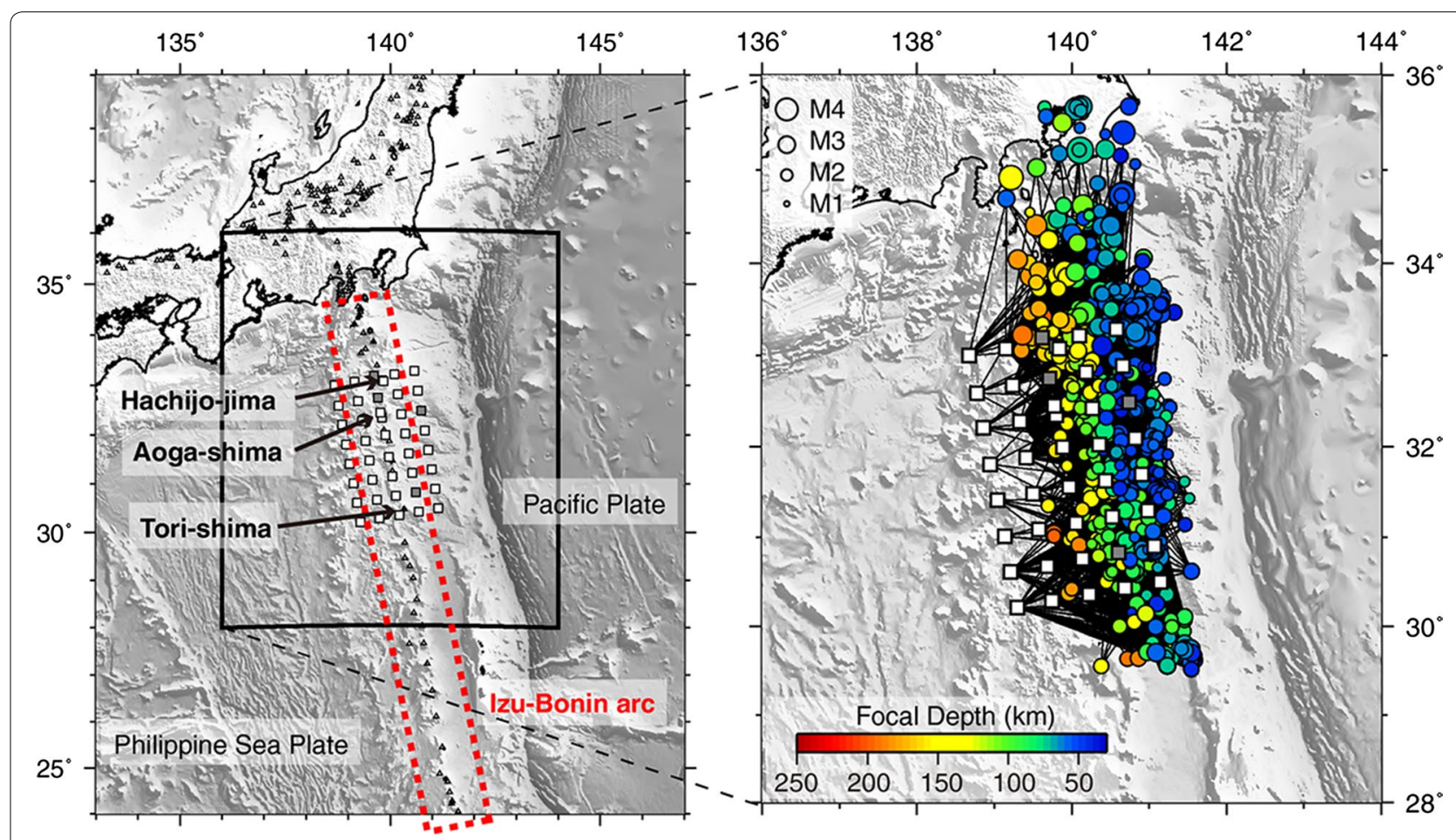

Fig. 1 Map view of the study area. White squares represent onshore seismic stations and OBSs used in this study, and gray squares are OBSs from which no data were available. Colored circles and black lines are epicenters of earthquakes and raypaths, respectively, used in this study

unclear, in contrast to the clear correlation of the crustal structures with volcanoes.

Another passive seismic imaging in northern IzuBonin arc that is an analysis of seismic wave scattering was conducted by Takahashi et al. (2011). They analyzed the $\mathrm{S}$-wave envelopes at $4-32 \mathrm{~Hz}$ and estimated the spatial variation of the power spectral density function (PSDF) of random velocity inhomogeneities that is a fractional fluctuation of seismic velocity from the average velocity. Their result showed strong inhomogeneities near the volcanic front around the crust $(0-30 \mathrm{~km}$ depth) and moderately strong inhomogeneities in the uppermost mantle beneath the volcanic front. Correlation of these moderately strong inhomogeneities with the volcano distribution was higher than that of seismic velocity. Their study assumed a PSDF which has powerlaw decay at large wavenumbers to take into account of frequency dependence of scattering and clarified these strong and moderately strong random inhomogeneities were commonly characterized by a gradual spectral gradient. This means that random inhomogeneities beneath the volcanoes are rich in short-wavelength spectra. They suggested that magma intrusions might account for the S-wave velocity fluctuations, but this interpretation would not explain the weak correlation with the seismic velocity structure.
In this study, we examined the S-wave attenuation tomography of the northern Izu-Bonin arc to elucidate the seismic structure of the crust and uppermost mantle. Seismic wave attenuation in this arc would be an important structural constraint to understand the current magma distribution and temperature structure. For the structure of random inhomogeneities, we used the results reported by Takahashi et al. (2011), and we applied the $\mathrm{S}$-wave attenuation tomography method of Takahashi et al. (2014) to the same seismic waveform data used by Takahashi et al. (2011). In this method, the maximum amplitudes at plural frequency bands are analyzed, and the attenuation structures, site amplification factors, and seismic source parameters are estimated simultaneously. The uncertainties of unknown parameters are evaluated by Monte Carlo sampling. In this paper, we outline the method and the seismic data and then describe the attenuation structure beneath the northern Izu-Bonin arc and its uncertainties. Finally, we examine the relations between $Q^{-1}$ and other seismic structures.

\section{Method}

It is assumed that the medium is characterized by random velocity fluctuations and inelasticity. The $\mathrm{S}$-wave velocity of the medium on the location $\mathbf{x}$ is set to $V(\mathbf{x})=V_{0}(1+\xi(\mathbf{x}))$, where $V_{0}$ and $\xi(\mathbf{x})$ are the 
background velocity and random fractional fluctuation, respectively. We imagine an ensemble of random media. The ensemble average of the fluctuations $\xi(\mathbf{x})$ satisfies $\langle\xi(\mathbf{x})\rangle=0$, where the angle brackets mean the ensemble average. If the characteristic scale of the random inhomogeneities is much larger than the wavelength of the incident wave, multiple forward scattering becomes dominant at large travel distances. Under this condition, envelopes of direct waves can be described by a Markov approximation of the parabolic wave equation (e.g., Sato 1989; Saito et al. 2002). The Markov approximation is a kind of stochastic interpretation of wave scattering in random media that gives the integration of forward scattering processes in thin layers from source to receiver (e.g., Williamson 1972). Takahashi et al. (2011) used this Markov approximation in their estimation of the random inhomogeneity structure in the northern Izu-Bonin arc. Here, we suppose that random inhomogeneities and $Q^{-1}$ vary in space. By using the Markov approximation, we can calculate the maximum amplitude for an impulsive wavelet radiated from a point source with a unit energy (see Saito et al. 2002 for details) that is equivalent to the scattering attenuation. Then, the $\mathrm{S}$-wave maximum amplitude $A_{\max }$ of root mean square (RMS) envelope in a narrow frequency band can be written as

$$
\begin{aligned}
A_{\max }(r, f)= & \frac{S(f)}{\sqrt{\rho V_{S}}} \cdot\left(\frac{1}{4 \pi r^{2}} \cdot F_{\text {scat }}(r, f, \xi(\mathbf{x})) \cdot F_{Q}\left(r, f, Q^{-1}(f, \mathbf{x})\right)\right)^{1 / 2} \\
& R_{\theta \phi} \sqrt{W_{\mathrm{s}}(f)},
\end{aligned}
$$

at travel distance $r$ and central frequency $f$. The three components on the right-hand side of Eq. (1) are, from left to right, the site effect, the path effect, and the source term.

The $\rho V_{S}$ is the $S$-wave impedance at the seismic station, where $V_{\mathrm{s}}$ and $\rho$ are, respectively, $S$-wave velocity and medium density at a seismic station. $S(f)$, the site amplification factor, is an unknown parameter in our inversion. It represents the local structure effect near a seismic station that cannot be assigned to the $S$-wave impedance or to the path effect. $S(f)$ includes the recording system response and boundary conditions of an OBS or onshore station, as well as the effect of local structure near the seismic station.

The path effect consists of geometrical spreading $1 / r$, scattering attenuation $F_{\text {scat }}(r, f, \xi(\mathbf{x}))$, and the $Q^{-1}$ effect. The $F_{\text {scat }}(r, f, \xi(\mathbf{x}))$ was evaluated for all raypaths and at all frequency bands by a numerical simulation with the Markov approximation (Takahashi et al. 2008). The attenuation factor $F_{Q}$ is set as

$$
F_{Q}\left(r, f, Q^{-1}(f, \mathbf{x})\right)=\exp \left(-2 \pi f \sum_{k} Q_{k}^{-1}(f, \mathbf{x}) \Delta r_{k} / V_{(2)}\right),
$$

where summation takes place along the raypath, $V_{0}$ is the $S$-wave velocity along the raypath, and $\Delta r_{k}$ is the path length of a segment. We assumed the frequency dependence of $Q^{-1}$ as

$$
Q^{-1}(f, \mathbf{x})=Q_{0}^{-1}(\mathbf{x}) \cdot f^{-\gamma(\mathbf{x})},
$$

where $Q_{0}^{-1}$ is $Q^{-1}$ at $1 \mathrm{~Hz}$. Even though parameter $\gamma$ and its spatial variation were not fully resolved in previous studies of attenuation tomography (e.g., Pozgay et al. 2009), this study set the $\gamma(\mathbf{x})$ as an unknown parameter with consideration for $\gamma$ variations in Japan (e.g., Carcole and Sato 2010).

The radiation pattern $R_{\theta \phi}$ in source term was set to the RMS average of $S$-wave radiation from a point shear-dislocation source $\bar{R}_{\theta \phi}=\sqrt{2 / 5}$. Source energy $W_{\mathrm{s}}$ is evaluated by using an omega-square source spectrum model as

$$
W_{\mathrm{s}}(f)=\frac{(2 \pi f)^{2}}{10 \pi \rho_{0} \beta_{0}^{5}}\left(\frac{M_{0}}{1+\left(f / f_{\mathrm{c}}\right)^{2}}\right)^{2},
$$

where $M_{0}$ and $f_{\mathrm{c}}$ are, respectively, the seismic moment and corner frequency and $\rho_{0}$ and $\beta_{0}$ are the density and $S$-wave velocity, respectively, in the source region (e.g., Kinoshita and Ohike 2002). We selected this source model to reduce the number of unknown parameters.

Unknown parameters in our attenuation tomography are $S(f), M_{0}, f_{\mathrm{c}^{\prime}}, Q_{0}^{-1}(\mathbf{x})$, and $\gamma(\mathbf{x})$. The study area was partitioned into $N$ blocks that are characterized by $Q_{0}^{-1}$ and $\gamma$. These parameters were estimated by minimizing the misfit and smoothness function $s$ :

$$
\begin{aligned}
s= & \sum_{\text {freq }} \sum_{\text {ray }}\left(\log \left(r A_{\max }^{\text {obs }}\right)-\log \left(r A_{\max }^{\text {calc }}\right)\right)^{2} \\
& +\frac{w_{Q_{0}}}{N}\left(\nabla \log Q_{0}^{-1}(\mathbf{x})\right)^{2}+\frac{w_{\gamma}}{N}(\nabla \gamma(\mathbf{x}))^{2} .
\end{aligned}
$$

The first term is the squared residual of the maximum amplitude $A_{\max }$ corrected for the geometrical spreading effect, and the second and third terms are constraint terms for the spatial smoothness of unknown parameters with weighting factors $w_{Q_{0}}$ and $w_{\gamma}$.

The inversion analysis consists of three steps to achieve a stable estimation of all parameters and a small tradeoff among unknown parameters (Takahashi 2012). The first step estimates the initial model by assuming a spatially uniform attenuation structure: $Q_{0}^{-1}(\mathbf{x})=Q_{0}^{-1}$ uni and $\gamma(\mathbf{x})=\gamma_{\text {uni. }}$. Only two site factors $S(f)$ are possible during this first step: one for onshore stations and another for OBSs. The second step estimates the $Q^{-1}(\mathbf{x})$ structure with $S(f)$ under the same constraint as in the first step. The third step refines $Q^{-1}(\mathbf{x})$ and the source parameters 
and also estimates $S(f)$ of all stations without any constraints for $S(f)$.

Source parameters were constrained as follows. During the first and second steps, we fixed the $M_{0}$ values of some moderate-sized earthquakes by using reference values from the moment tensor catalog, which is routinely published on the Web site of the broadband F-net seismic network (Okada et al. 2004). We further constrained energy radiation at frequencies higher than $f_{\mathrm{c}}$ by assuming Brune's stress drop (Brune 1970) $\Delta \sigma=(7 / 16)\left(M_{0} /\left(2.34 \beta_{0} / 2 \pi f_{\mathrm{c}}\right)^{3}\right)$ for the referenced events to be $1 \mathrm{MPa}$ during the first and second steps. Stress drops of other earthquakes were restricted to a narrow range $(0.1-10 \mathrm{MPa})$ during the first and second steps. In the third step, we set a sufficiently wide sampling range of 0.01-100 MPa for all earthquakes. We also set broad sampling ranges for the unknown parameters as follows: $10^{10} \mathrm{Nm}<M_{0}<10^{18} \mathrm{Nm} ; 10^{-4}<Q_{0}^{-1}<10^{-1}$; $-0.5<\gamma<2.0$; and $1 / 1000<S(f)<1000$.

Solutions and uncertainties of this inverse problem were evaluated by the exchange Monte Carlo (EMC) method (Hukushima and Nemoto 1996), which is a Markov chain Monte Carlo (MCMC) method. The EMC sets plural temperatures $\tau_{i},\left(i=1-n_{\tau}\right)$ and generates random number sequences by MCMC for all $\exp \left(-s / \tau_{i}\right)$ in parallel. If a sampling point at a higher temperature $\tau_{j+1}$ has smaller $s$ than that at a lower temperature $\tau_{j}$, the sampling point at $\tau_{j+1}$ will be exchanged with the point at $\tau_{j}$. The exp $\left(-s / \tau_{i}\right)$ with larger $\tau_{i}$ becomes smooth and gradual and therefore ensures parameter sampling over the whole parameter space. In this study, we set temperatures $\tau_{i}$ as

$$
\tau_{i}=\left\{\begin{array}{ll}
0.01 \times 1.3^{i-1} & (1 \leq i \leq 10) \\
0.01 \times 1.3^{9} \times 1.8^{i-10} & (11 \leq i \leq 24)
\end{array} .\right.
$$

If we consider the Gaussian likelihood function, uncertainties of unknown parameters are evaluated by the standard deviations of the sampled points at $\tau_{i} \leq 2 \sigma^{2}$, where $\sigma^{2}$ is the variance of $\log \left(r A_{\max }\right)$. This standard deviation is usually called the Monte Carlo standard error. We estimated the optimal solution by taking the ensemble average of the sampled points at $\tau_{1}$, and its variance $\sigma^{2}$ from the squared residuals of $\log \left(r A_{\max }\right)$ at $\tau_{1}$. These results and errors were evaluated by using 5000 models picked every 100 iterations. This uncertainty reflects the quality and quantity of observed data and therefore would give a clear insight on the reliability of inversion result.

\section{Observations and data}

Seismic observation in the northern Izu-Bonin arc (Obana et al. 2010) was conducted from April 2006 to
July 2006 with 40 OBSs. Each OBS was equipped with a three-component $4.5 \mathrm{~Hz}$ seismograph. Waveform data were recorded with a $100 \mathrm{~Hz}$ sampling rate. After the observation period, 37 OBSs were recovered, and 36 of these (white squares in Fig. 1) had successfully recorded ground motion. In this area, the National Research Institute for Earth Science and Disaster Prevention (NIED) of Japan (Okada et al. 2004; Obara et al. 2005) operates some seismic stations, two on Hachijo-jima and one on Aoga-shima. Seismic data recorded at these stations were also used in this study. Seismic data analysis with both of OBSs and onshore stations detected approximately 4500 earthquakes. Obana et al. (2010) determined the 1-D velocity structures of $V_{\mathrm{p}}$ and $V_{\mathrm{s}}$ and then estimated the initial hypocenter locations using the hypomh program (Hirata and Matsu'ura 1987) before travel time tomography. Following the random inhomogeneity study of Takahashi et al. (2011), we used some of these initial hypocenter locations to estimate the random inhomogeneity structure, and we also referred to the unified hypocenter list provided by the Japan Meteorological Agency (JMA) for events occurring north of the study area $\left(>34.5^{\circ} \mathrm{N}\right)$.

The maximum $\mathrm{S}$-wave amplitudes were measured from the RMS envelopes at 4-8, 8-16, and 16-32 Hz. RMS envelopes were composed of the horizontal components of the velocity seismograms $v_{H 1}(t)$ and $v_{H 2}(t)$ as $\sqrt{v_{H 1}^{2}(t)+v_{H 2}^{2}(t)}$. We applied the moving average for envelopes in a window whose length was twice the central period. We measured the maximum amplitude in a 30-s time window starting from the S-wave onset. Examples of observed waveform and envelopes used in this work were shown in Figure 4 in Takahashi et al. (2011). The time window length was manually shortened for the case that large amplitude waves were observed after S-wave peak arrival. Some envelopes with no clear peak arrival because of strong scattering or absorption were excluded. Seismograms of these excluded data usually show clear S-wave at lower frequency, but weak or no $\mathrm{S}$-wave signals at higher frequency. Most of them were the records of backarc side stations. These excluded data suggest existences of high $Q^{-1}$ or strong inhomogeneity in the backarc, but this information cannot be quantified as attenuation or random inhomogeneity structure.

The earthquakes selected for this analysis occurred near the subducting Pacific plate at $35-300 \mathrm{~km}$ depth, and their maximum magnitude was 4.7 . The hypocentral distance ranged from 100 to $300 \mathrm{~km}$. These criteria were introduced in the peak delay time analysis of Takahashi et al. (2011) to satisfy some requirements in the Markov approximation. For example, the minimum travel distance was set to secure a sufficient accumulation of multiple forward scattering. Numbers of input data for 
tomography are $6107(4-8 \mathrm{~Hz}), 6138(8-16 \mathrm{~Hz})$, and 4748 $(16-32 \mathrm{~Hz})$. Seismic raypaths were evaluated under the $1-D$ velocity structure used by JMA for the unified hypocenter list (Ueno et al. 2002) as with the study of random inhomogeneities (Takahashi et al. 2011). The study area was divided into 650 blocks, whose dimensions were either $0.60^{\circ} \times 0.60^{\circ}$ horizontally $\times 30 \mathrm{~km}$ thick $(0-30 \mathrm{~km}$ depth $)$ or $0.30^{\circ} \times 0.30^{\circ}$ horizontally $\times 20 \mathrm{~km}$ thick ( $>30 \mathrm{~km}$ depth). The number of earthquakes for these data was 651 . The number of referenced $M_{0}$ values was 20. We set different impedances for OBSs and island stations. For the OBSs, we used $S$-wave velocity and density values for ocean bottom sediment, $V_{\mathrm{s}}=0.15 \mathrm{~km} / \mathrm{s}$ and $\rho=2.1 \mathrm{~g} / \mathrm{cm}^{3}$, taking into account the waveform modeling of OBS data in the northern Izu-Bonin $\operatorname{arc}$ (Sato et al. 2009). For the island stations, we used $V_{\mathrm{s}}=3.15 \mathrm{~km} / \mathrm{s}$ and $\rho=2.7 \mathrm{~g} / \mathrm{cm}^{3}$ (Takahashi et al. 2014). We set $V_{0}$ to $4 \mathrm{~km} / \mathrm{s}, \rho_{0}=3.2 \mathrm{~g} / \mathrm{cm}^{3}$ and $\beta_{0}=4.4 \mathrm{~km} / \mathrm{s}$, following Takahashi (2012).

\section{Results}

In the first analysis step, uniform $Q^{-1}$ was estimated to be $Q^{-1}(f)=10^{-2.8} f^{-0.68}$. This value indicates a relatively weak attenuation of the S-wave $Q^{-1}$ and is similar to the uniform $Q^{-1}$ estimated in southwestern Japan, $Q^{-1}$ $(f)=10^{-2.7} f^{-0.67}$ (Takahashi et al. 2014).

Weighting factors were selected by considering the L-curves (Fig. 2a, b) (e.g., Hansen and O'Leary 1993) of the smoothness constraints. The L-curve is a plot of smoothness constraints against the RMS residual of $\log \left(r A_{\max }\right)$, and it clarifies adequate smoothness for sufficiently small residuals of $\log \left(r A_{\max }\right)$. Although both L-curves show large scatter, we identified a corner of the L-curve for $\gamma$ at $\log \left(r A_{\max }\right)=0.19-0.20$ (Fig. 2b, red arrow). The L-curve for $Q_{0}^{-1}$ (Fig. 2a) does not show any clear corners, but smoothness tended to increase with decreasing the residual of $\log \left(r A_{\max }\right)$ when the residual was smaller than $0.20-0.22$. Here, we assumed an optimal RMS residual of 0.20 . With this residual, the smoothness of $Q_{0}^{-1}$ should be in the range $1.0 \times 10^{-4}-1.0 \times 10^{-3}$ and that of $\gamma$ should be $1.0 \times 10^{-4}-1.0 \times 10^{-3}$. By plotting these smoothness constraints against their weighting factors (Fig. 2c, d), we inferred that both $w_{Q_{0}}$ and $w_{\gamma}$ should be $5.0 \times 10^{3}-1.0 \times 10^{4}$; thus, we selected $w_{Q_{0}}=1.0 \times 10^{4}$ and $w_{\gamma}=1.0 \times 10^{4}$. Using these weighting factors, we evaluated the Monte Carlo standard
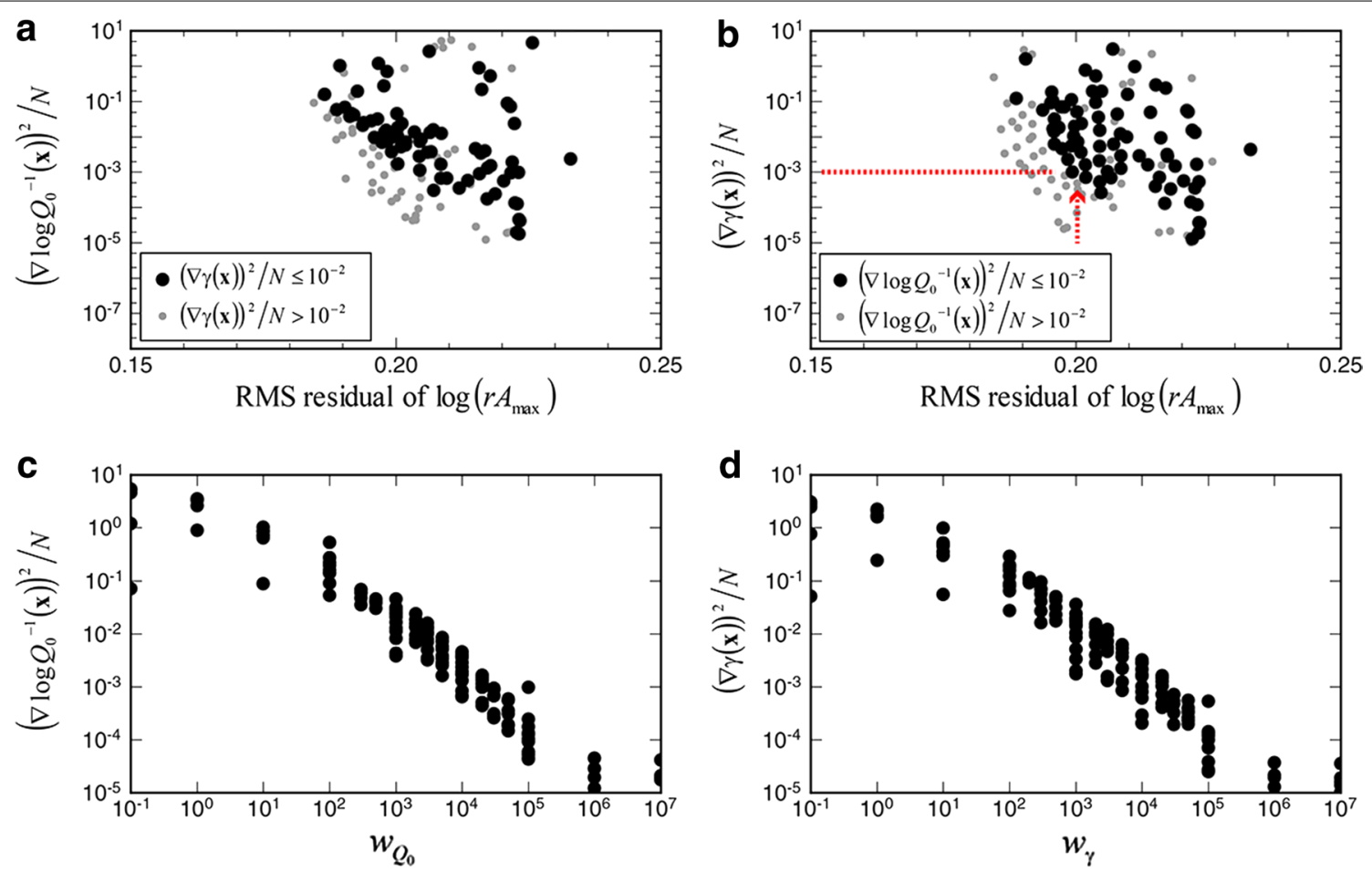

Fig. 2 a L-curve plot of $\left(\nabla \log Q_{0}^{-1}(\mathbf{x})\right)^{2} / N$. Black and gray symbols reflect the smoothness of $\gamma$. $\mathbf{b} L$-curve plot of $(\nabla \gamma(\mathbf{x}))^{2} / N$. Black and gray symbols reflect the smoothness of $Q_{0}^{-1}$. The red arrow and dotted line indicate the corner of the $L$-curve that we chose in this study. $\mathbf{c}$ Plot of $\left(\nabla \log Q_{0}^{-1}(\mathbf{x})\right)^{2} / N$ against $w_{Q_{0}} \mathbf{d}$ Plot of $(\nabla \gamma(\mathbf{x}))^{2} / N$ against $w_{\gamma}$ 
errors by using samples at $\tau_{1}-\tau_{9}\left(\tau_{9}=0.0816\right)$; here, $\tau_{9}$ is close to $2 \sigma^{2}(\sim 0.08)$. Note that large scatter of L-curve is due to mutual interaction between $w_{Q_{0}}$ and $w_{\gamma}$. For example, if $w_{\gamma}$ is set in a narrow range near the optimal value, the L-curve for $\gamma$ shows smaller scatter.

We next examined the relationship between the source parameters $M_{0}$ and $f_{\mathrm{c}}$ (Fig. 3). Most of the estimated $M_{0}$ were distributed in a narrow range from $10^{12}$ to $10^{14} \mathrm{Nm}$. The smallest $M_{0}$ of the referenced events was $2.26 \times 10^{14}$ Nm. $M_{0}$ of unreferenced events would be smaller than this minimal moment; therefore, this moment range seems reasonable. Stress drop was scattered around 1-10 MPa. This stress drop tends to be larger than that reported by previous studies (e.g., Takahashi et al. 2014), but larger stress-drop events tend to show larger Monte Carlo standard errors of $f_{\mathrm{c}^{\prime}}$. A possible origin of larger uncertainty for higher $f_{\mathrm{c}}$ is small number of data at the highest frequency band $(16-32 \mathrm{~Hz})$, since accurate estimation of higher $f_{\mathrm{c}}$ requires seismic data at higher frequency.

The site factor $S(f)$ (Fig. 4a) generally decreased with increasing frequency, and $S(f)$ in each frequency band was distributed randomly in space without any systematic trend. The Monte Carlo standard errors (Fig. 4b) were generally small, with some exceptions. The large error in the backarc side at $16-32 \mathrm{~Hz}$ was probably due to the small number of data. Except at this station, site amplification factors were distributed from 0.04 to 2.3. The decreasing $S(f)$ with increasing frequency

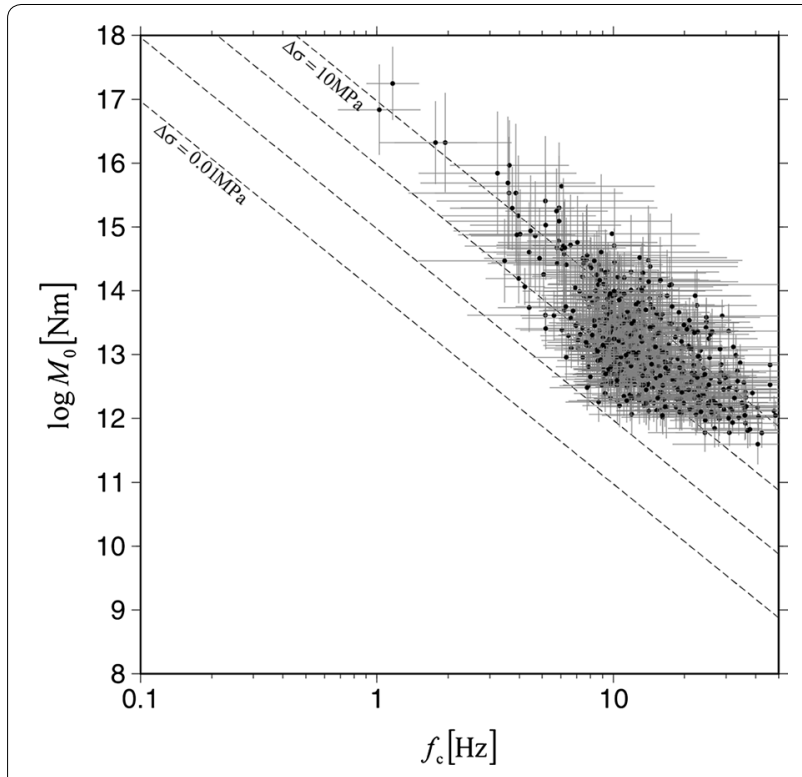

Fig. 3 Plot of seismic moment $M_{0}$ against the corner frequency $f_{c}$. Error bars represent the Monte Carlo standard errors. Dashed lines represent constant values of Brune's stress drop can be interpreted to mean that $Q^{-1}$ in the sediment layer beneath the seafloor was frequency independent or only weakly frequency dependent. Most site factors were less than 1.0, which means that attenuation occurred in the vicinity of seismic stations. The small site amplification factors may mean that the assumed impedances at the seismic stations were smaller than the true values.

The 3-D attenuation structures and the Monte Carlo standard errors are shown in map views of the results at $0-30,30-50$, and $50-70 \mathrm{~km}$ depths (Figs. 5, 6, 7). Figure 5 shows $Q_{0}^{-1}$ and $\gamma$, and their Monte Carlo standard errors; for each depth interval, Figs. 6 and 7 show $Q^{-1}$ values and their Monte Carlo standard errors, respectively, at $4-8,8-16$, and $16-32 \mathrm{~Hz}$. $Q^{-1}$ values were calculated from the estimated $Q_{0}^{-1}$ and $\gamma$ on the basis of a simple relation (Eq. 3). Examination of these $Q^{-1}$ maps can give intuitive insights into the attenuation structures. By applying the Born approximation to the random inhomogeneities in this area as with Takahashi (2012), we confirmed that $Q^{-1}$ of backscattering was less than $3 \%$ of the estimated $Q^{-1}$ in the study area. Thus, we consider the estimated $Q^{-1}$ to mainly reflect the inelasticity of medium.

At $0-30 \mathrm{~km}$ depth, both $Q_{0}^{-1}$ and $\gamma$ showed clear differences between the northern and southern parts of the study area, but $Q^{-1}$ was nearly constant. The Monte Carlo standard error of $Q^{-1}$ was large only on the forearc side. This large error was due mainly to the large uncertainty of $Q_{0}^{-1}$. We note that inversion results with smaller $w_{Q_{0}}$ and $w_{\gamma}$ frequently showed lower $Q^{-1}$ on the forearc side than on the backarc side. Therefore, our data may contain information on the $E-W$ variation of $Q^{-1}$ in this depth range, but there is not enough information in our data to resolve such horizontal variation. Although the $\mathrm{N}-\mathrm{S}$ variations of $Q_{0}^{-1}$ and $\gamma$ should be reliable, it is difficult to infer their possible origins because they show no clear correlation with other seismic structures.

At $30-50$ and $50-70 \mathrm{~km}$ depth, $Q^{-1}$ showed clear variations between the area east and west of the volcanic front. At $4-8 \mathrm{~Hz}, Q^{-1}$ was about $10^{-2.3}-10^{-2.5}$ in high$Q^{-1}$ areas (A, B, C and D in Fig. 6) and $\sim 10^{-3.2}$ at most on the forearc side. In both the northern and southern parts of the study area, the standard errors of $Q_{0}^{-1}$ were larger on the forearc side. Because the number of data on the forearc side was larger than that on the backarc side, the larger errors may reflect the uncertainties of the hypocenter locations outside the network. At $30-50 \mathrm{~km}$ depth, we recognized two high- $Q^{-1}$ areas beneath the volcanic front. One is the area-A beneath Hachijo-jima and another is area-B beneath Tori-shima. The $Q^{-1}$ values in both high- $Q^{-1}$ areas are up to $10^{-2.50}$ at $8-16 \mathrm{~Hz}$. 


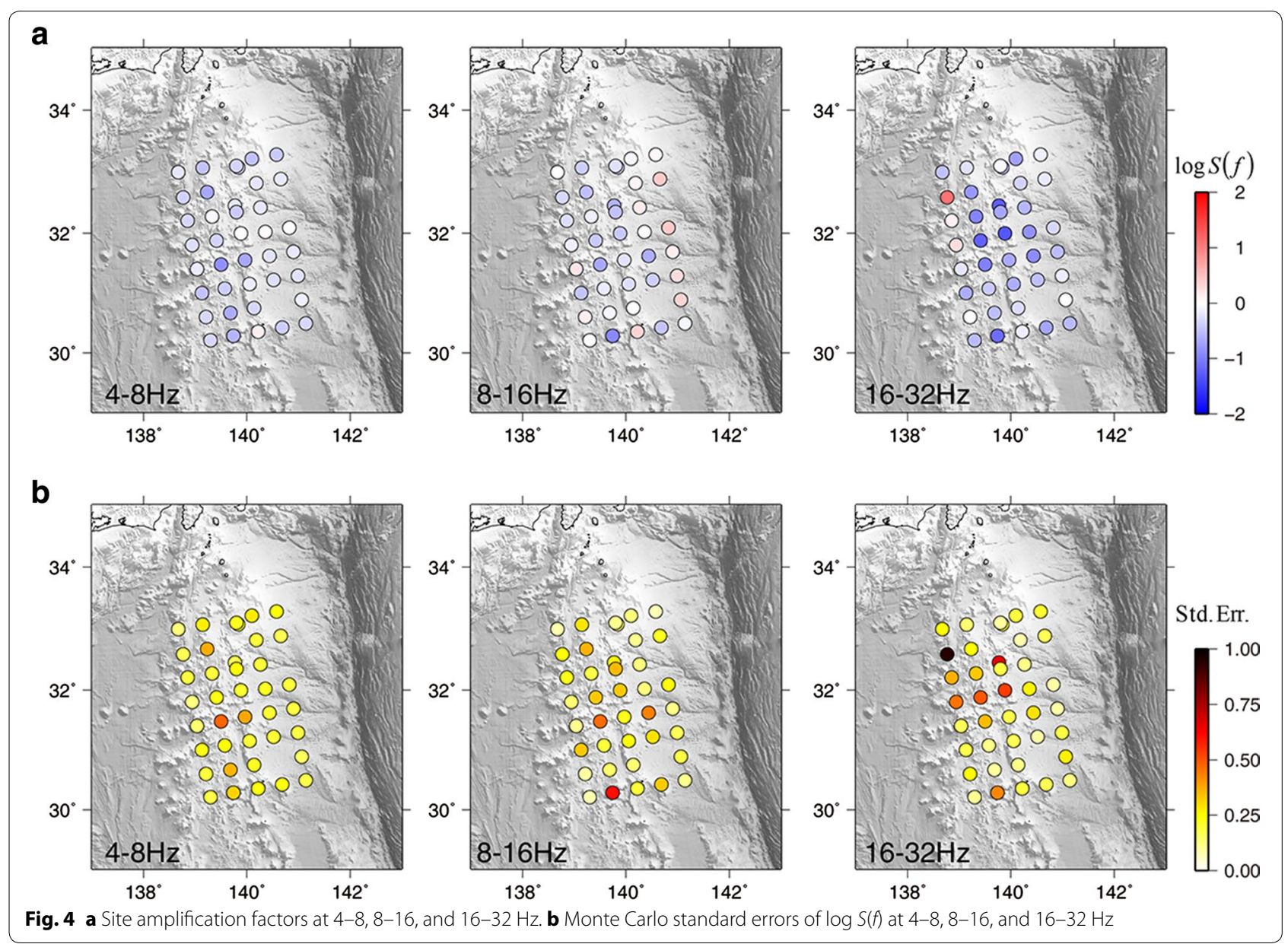

The Monte Carlo standard errors showed that this alongarc variation is reliable. Note that results with smaller weights $\left(w_{Q_{0}}=w_{\gamma}=1.0 \times 10^{3}\right)$ showed third high- $Q^{-1}$ $\left(\sim 10^{-2.5}\right.$ at $\left.8-16 \mathrm{~Hz}\right)$ anomaly on the backarc side of Sumisu-jima. However, this anomaly was not significant due to large uncertainty. At $50-70 \mathrm{~km}$ depth, two high- $Q^{-1}$ areas were also imaged beneath the volcanic front at areas $C$ and D. $Q^{-1}$ at $8-16 \mathrm{~Hz}$ in $C$ and $\mathrm{D}$ are up to $10^{-2.28}$ and $10^{-2.47}$, respectively. They are at different locations from the high- $Q^{-1}$ areas (A and B) at $30-50 \mathrm{~km}$ depth. North of Hachijo-jima at $50-70 \mathrm{~km}$ depth, however, $Q^{-1}$ showed large uncertainties. Therefore, the meaningful difference between $Q^{-1}$ in these two depth intervals is the width of the low- $Q^{-1}$ area between the two high- $Q^{-1}$ areas. We also recognized moderately high- $Q^{-1}$ areas in the north and south on the forearc side (E and F in Fig. 6) that is the most evident at $4-8 \mathrm{~Hz} . Q^{-1}$ in the northern anomaly is $10^{-3.0}$ at $4-8 \mathrm{~Hz}$, and that in southern anomaly is up to $10^{-2.75}$ at $4-8 \mathrm{~Hz}$. The anomaly in the northern part was not significant owing to the large uncertainty, but the southern anomaly is worth discussing.

\section{Discussion}

Along-arc variation of $Q^{-1}$ was clearly imaged in the uppermost mantle at $30-50 \mathrm{~km}$ depth beneath the volcanic front. However, its correlation with the volcano distribution was not high because of the large horizontal extent of the high- $Q^{-1}$ areas. The seismic velocity structure (Obana et al. 2010) shows two low-Vs anomalies at $30-70 \mathrm{~km}$ depth beneath the volcanic front: One is north of Aoga-shima and the other is south of Sumisujima. Most of the high- $Q^{-1}$ areas at $30-50$ and $50-70 \mathrm{~km}$ depth are within these low-Vs areas, and lower $Q^{-1}$ beneath the volcanic front was imaged between Aogashima and Sumisu-jima (Fig. 8). Accordingly, low-Vs and high $Q^{-1}$ were mostly correlated in this arc. According to laboratory experiments, $\log Q^{-1}$ can be represented as $\log Q^{-1}=\gamma(X-Y / T)$, where $X$ and $Y(>0)$ are parameters related to rock properties and pressure and $T$ is medium temperature (e.g., Karato and Jung 1998; Behn et al. 2009). In this entire study area, $\gamma$ was positive, and $\gamma$ in high- $Q^{-1}$ areas tended to be smaller than on the forearc side. Although this horizontal variation of $\gamma$ means that high $Q^{-1}$ beneath volcanoes did not 
a
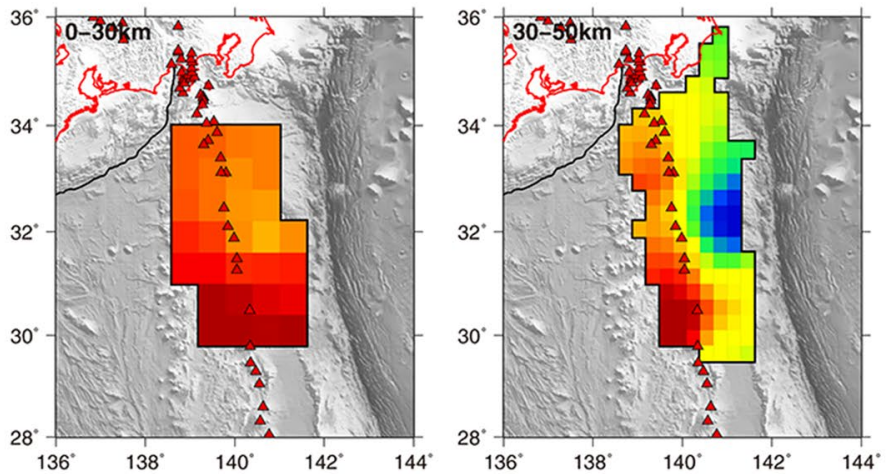

b

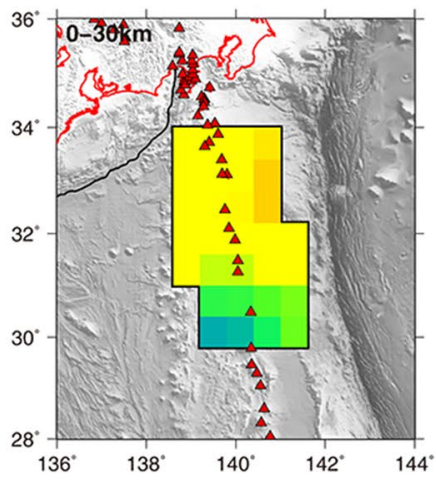

C
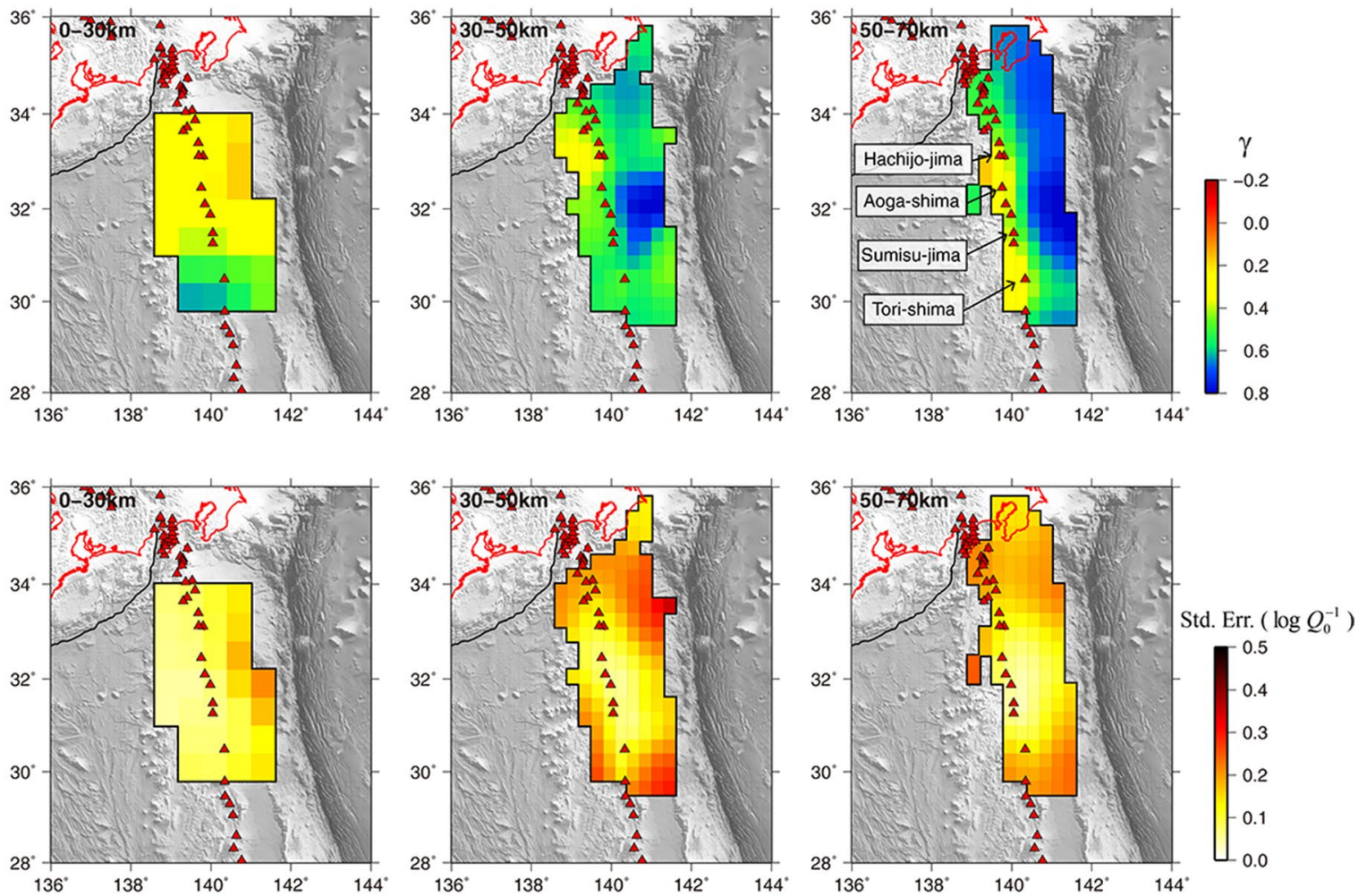

d
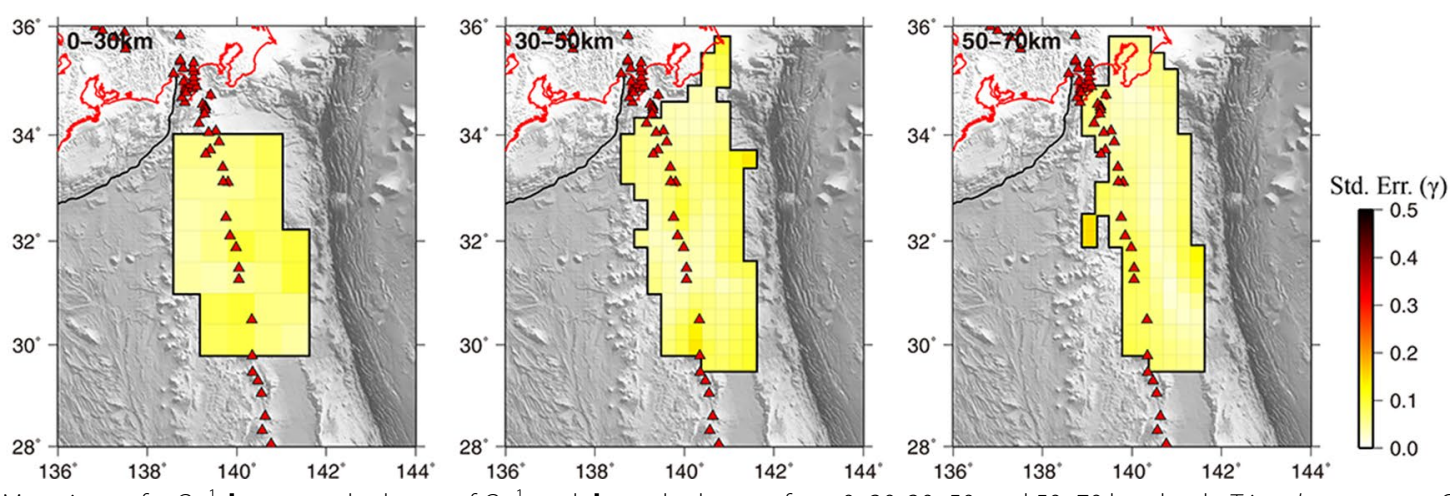

Fig. 5 Map views of $\mathbf{a} Q_{0}^{-1}, \mathbf{b} \gamma, \mathbf{c}$ standard error of $Q_{0}^{-1}$, and $\mathbf{d}$ standard error of $\gamma$ at $0-30,30-50$, and $50-70 \mathrm{~km}$ depth. Triangles represent Quaternary volcanoes
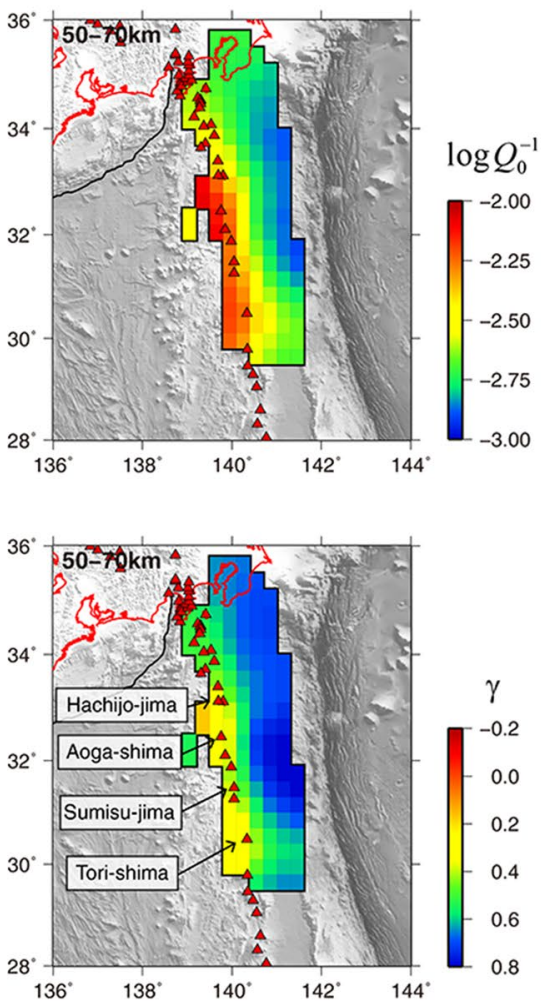


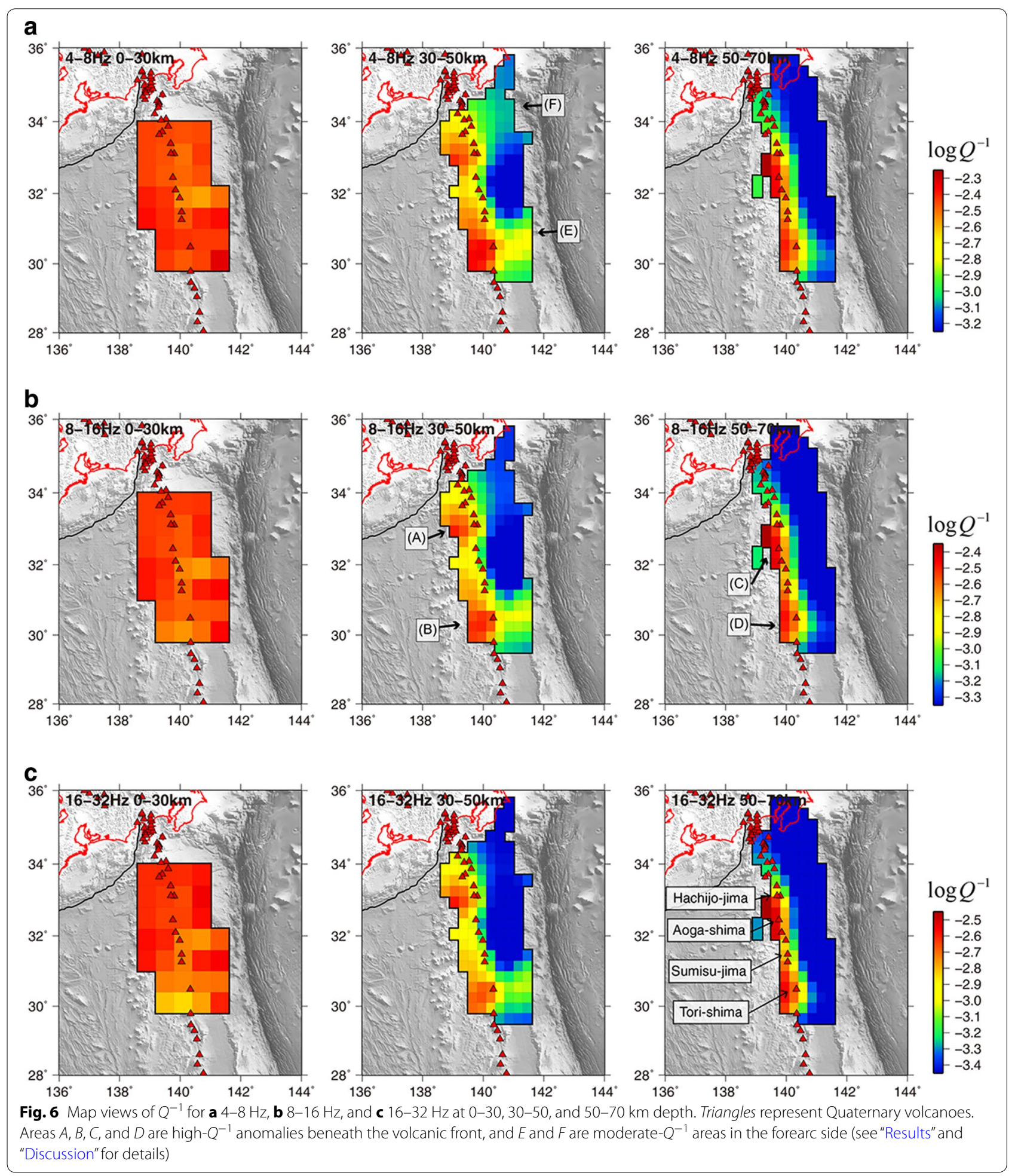

necessarily correspond to high temperatures, $\gamma$ showed very weak variation near Tori-Shima. This result suggests that the high $Q^{-1}$ beneath Tori-shima (area-B) at $30-50 \mathrm{~km}$ depth is probably an indicator of higher temperature there than on the forearc side, and the moderate $Q^{-1}$ between Hachijo-jima and Tori-shima likely means lower temperatures than in the Tori-shima area. The high- $Q^{-1}$ area in the low-velocity anomaly beneath 


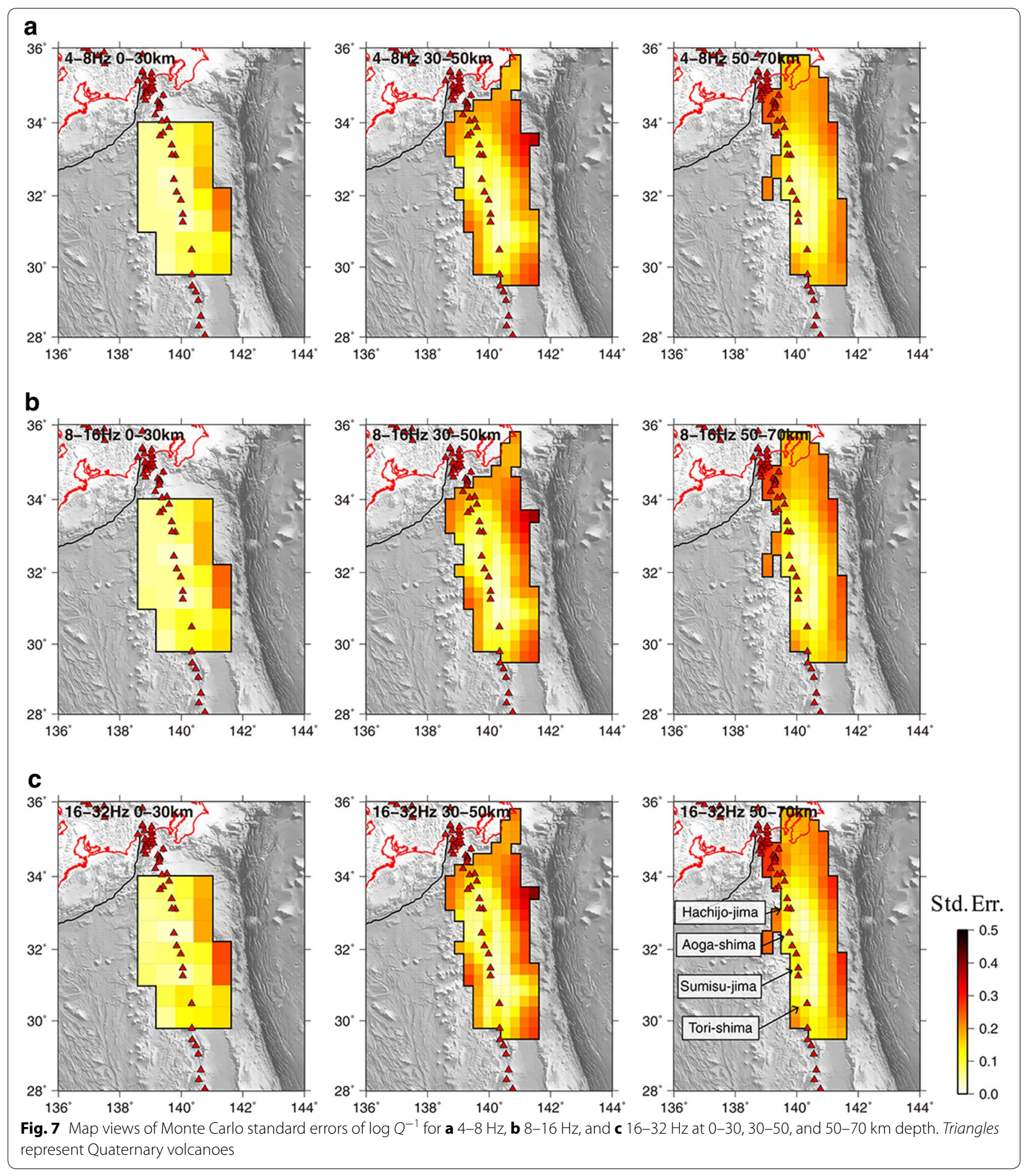

Hachijo-jima may indicate high temperature, as in ToriShima area, but because of the small $\gamma$ there, we cannot be certain. It is reasonable to infer that high- $Q^{-1}$ and low-velocity areas are the most likely candidates for high-temperature areas, but this possibility can only be confirmed by a detailed examination with suitable values for $X$ and $Y$ beneath Hachijo-jima. Tamura et al. (2002) hypothesized hot fingers of the mantle wedge were present in northeastern Japan, on the basis of the correlation between seismic velocity in the mantle wedge and the 


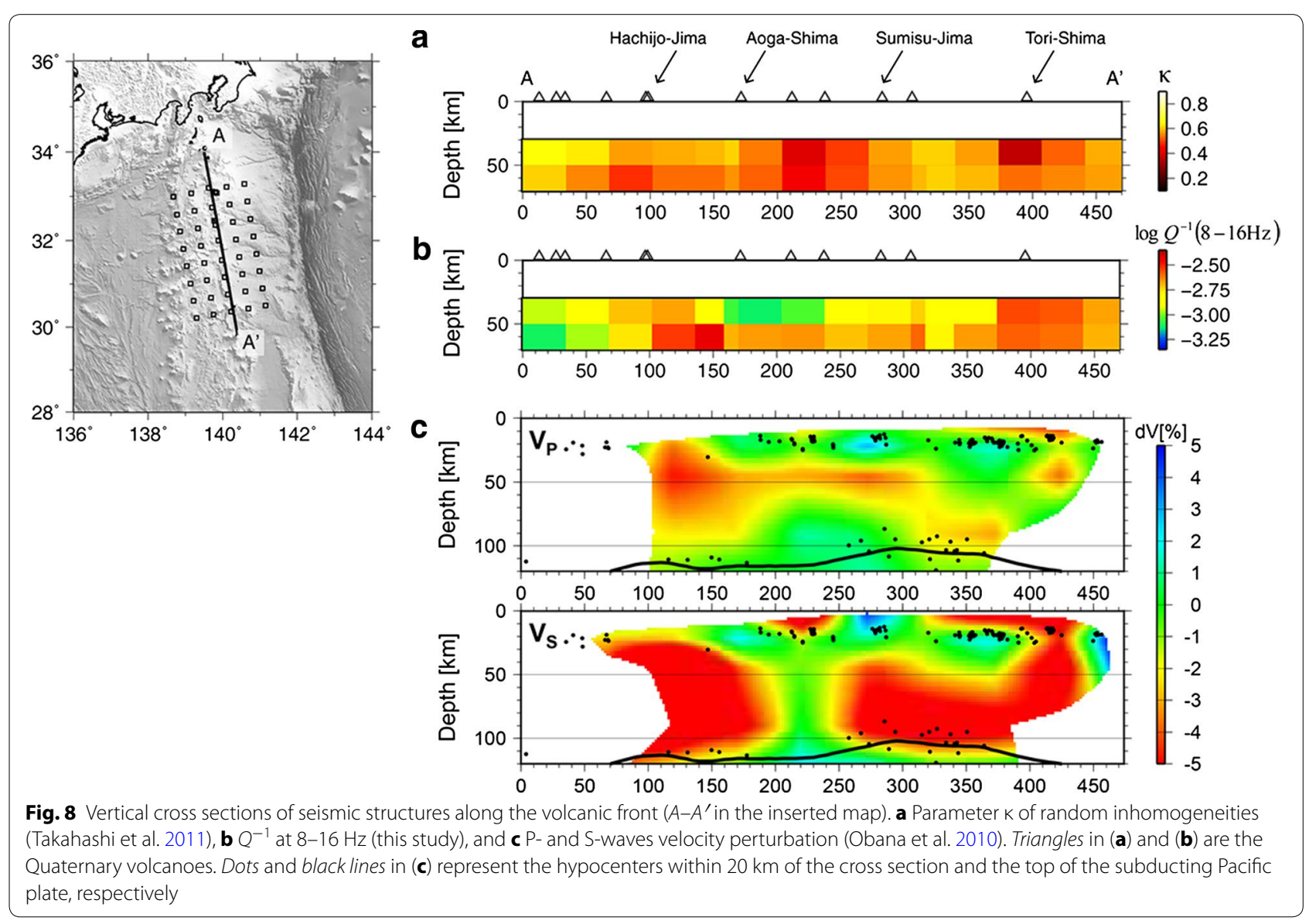

Quaternary volcano distribution. In the northern IzuBonin arc, we therefore might expect two hot regions in the uppermost mantle to be located in the high- $Q^{-1}$ areas.

Anomalies of random inhomogeneities at $30-70 \mathrm{~km}$ depth in Takahashi et al. (2011) were imaged beneath Hachijo-jima, the Aoga-shima-Sumisu-jima area, and Tori-shima. This random inhomogeneity structure was estimated by assuming the von Kármán type power spectral density function (PSDF) $P(m)=8 \pi^{3 / 2} \varepsilon^{2} a^{3} \Gamma(\kappa+3 / 2)\left(1+a^{2} m^{2}\right)^{-(\kappa+3 / 2)} / \Gamma(\kappa)$, where $\gamma$ is the gamma function, $m$ is the wavenumber in space, and parameters $a, \varepsilon$, and $\kappa$ are, respectively, the correlation distance, the root mean square of fractional fluctuation, and a parameter related to the spectral gradient at large wavenumbers $(m \gg 1 / a)$. The three anomalous random inhomogeneities were commonly characterized by smaller $\mathrm{\kappa}$ (Fig. $8 \mathrm{a}$ ) and in these areas $P(m)$ at large wavenumbers were moderately large compared with the surrounding areas. In these small $k$ areas, random inhomogeneities are rich in short-wavelength spectra. Takahashi et al. (2011) interpreted these moderately strong inhomogeneities to reflect magma inclusions, because fluid magma can cause large $\mathrm{S}$-wave velocity fluctuations. However, recent studies in southwestern Japan (Takahashi et al. 2013, 2014) have shown that strong inhomogeneities with a gradual spectral gradient show high spatial correlations with volcanic rock areas rather than with the Quaternary volcano distribution and that the attenuation structure in southwestern Japan seems to be related to magma and fluid distributions. Therefore, the three areas of anomalies of random inhomogeneities in the uppermost mantle reflect the accumulated intrusions of volcanic rocks during volcanic activity, whereas high- $Q^{-1}$ areas probably reflect the current magma distribution or high-temperature regions. The accumulated volcanic processes would be more relevant to the generation of arc volcanoes and continental crust in this arc. We have already inferred the presence of two hot regions in high- $Q^{-1}$ areas in the uppermost mantle. However, we may need to consider three hot regions at the top of uppermost mantle to describe the arc evolution and volcano distribution. Tamura et al. (2009) suggested that horizontal dykes in the crust could explain small spatial-scale variation of the middle crust on the basis of crustal structures, seismic reflectors, 
and petrological information. Our interpretation of the random inhomogeneities suggests the importance of volcanic processes in the current low- $Q^{-1}$ area in the uppermost mantle.

The forearc side of the mantle wedge showed moderately high- $Q^{-1}$ areas at $30-50 \mathrm{~km}$ depth at areas $\mathrm{E}$ and F. Taking into account the uncertainty, only the anomaly in the south (area-E) is meaningful. Travel time tomography imaged some small low $-V_{\mathrm{p}}$ and low- $V_{s}$ anomalies on the forearc side of the volcanic front, $50 \mathrm{~km}$ east of Aoga-shima, Sumisu-jima, and Tori-shima in the crust and uppermost mantle (Fig. 9 in Obana et al. 2010). They argued that the anomalies were related to the frontal arc high, which is a remnant Oligocene arc. Our moderately high- $Q^{-1}$ area included only one low-velocity anomaly, east of Tori-shima. Therefore, the results of the present study suggest that this $Q^{-1}$ anomaly is not necessarily due to the remnant arc. The east side of this moderately high$Q^{-1}$ area is in the subducting Pacific plate, and $Q^{-1}$ at 16-32 Hz showed a slight increase on the eastern side of this $Q^{-1}$ anomaly. Even though this increase of $Q^{-1}$ may not be significant given the uncertainties, it is possible that this moderately high $Q^{-1}$ was artificially generated by connecting the high- $Q^{-1}$ area beneath Tori-shima with one in the subducting slab. If a $Q^{-1}$ anomaly exists in the subducting slab in this area, it would have important implications for studies on fluid transportation and arc magmatism beneath this arc. In southwestern Japan, slight increases of $Q^{-1}$ were found near the top of the subducting slab (Takahashi et al. 2014). Even though the spatial resolution was too low for a detailed examination, $Q^{-1}$ would be a sensitive indicator of fluid distribution in the subducting plate when we remove the apparent attenuation effect due to multiple scattering. Studies of $Q^{-1}$ using data from OBSs near the trench axis or on the incoming plate would clarify possible origins of $Q^{-1}$ near the subducting plate.

\section{Conclusion}

This study estimated the $Q^{-1}$ attenuation structure in the northern Izu-Bonin arc, taking into consideration the apparent amplitude attenuation due to random velocity inhomogeneities. At $0-30 \mathrm{~km}$ depth, the inversion result showed large Monte Carlo standard errors on the forearc side, which prevented horizontal variations of $Q^{-1}$ from being resolved. Meanwhile, at $30-70 \mathrm{~km}$ depth, we clearly imaged some anomalies of high and moderate $Q^{-1}$ beneath the volcanic front and in the forearc side. Beneath the volcanic front, two high- $Q^{-1}$ zones were almost colocated with low-velocity anomalies. These high- $Q^{-1}$ areas are the most likely candidates for high-temperature regions beneath the volcanoes. The studies on wave scattering had reported that random inhomogeneities showed three anomalies at $30-70 \mathrm{~km}$ depth beneath the volcanic front (Takahashi et al. 2011). All of these anomalies of random inhomogeneities were characterized by a gradual spectral gradient of power spectral density at large wavenumber. If we consider relatively high correlation between such random inhomogeneity with gradual spectral gradient and igneous rock area that is recently pointed out in the southwestern Japan (Takahashi et al. 2013), the most probable interpretation of such spectra is the existence of volcanic rock, related to the accumulated magma intruded during episodes of volcanic activity. Therefore, the different distributions of $Q^{-1}$ and random inhomogeneities imply temporal changes of volcanic activity in this arc.

\section{Abbreviations}

OBS: Ocean bottom seismograph; PSDF: Power spectral density function; RMS: Root mean square; EMC: Exchange Monte Carlo; MCMC: Markov chain Monte Carlo; JMA: Japan Meteorological Agency; NIED: National Research Institute for Earth Science and Disaster Prevention.

\section{Authors' contributions}

TT estimated and interpreted the $Q^{-1}$ structure and drafted the paper with contributions from all co-authors. $\mathrm{KO}$ participated in data acquisition as a chief scientist. SK organized the data-acquisition cruises and participated in the interpretation. All authors contributed to scientific discussions. All authors read and approved the final manuscript.

\section{Acknowledgements}

This work was partially supported by Grant-in-Aid for Young Scientists (B) (20740261) and Grant-in-Aid for Scientific Research (B) (20340122). We thank those at NIED involved in the development and ongoing maintenance of the Hi-net and F-net systems. Generic Mapping Tools software (Wessel and Smith 1998) was used to produce the figures presented here, and Seismic Analysis Code software (Goldstein et al. 2003) was used for signal processing.

\section{Competing interests}

The authors declare that they have no competing interests.

Received: 21 December 2015 Accepted: 22 March 2016

Published online: 04 April 2016

\section{References}

Behn MD, Hirth G, Elsenbeck JR II (2009) Implications of grain size evolution on the seismic structure of the oceanic upper mantle. Earth Planet Sci Lett 282:178-189. doi:10.1016/j.epsl.2009.03.014

Brune JN (1970) Tectonic stress and the spectra of seismic shear waves from earthquakes. J Geophys Res 75:4997-5009

Carcole E, Sato H (2010) Spatial distribution of scattering loss and intrinsic absorption of short-period $\mathrm{S}$ waves in the lithosphere of Japan on the basis of the Multiple Lapse Time Window Analysis of Hi-net data. Geophys J Int 180:268-290. doi:10.1111/j.1365-246X.2009.04394.x

Goldstein P, Dodge D, Firpo M, Lee M (2003) SAC2000: Signal processing and analysis tools for seismologists and engineers. In: Lee WHK, Kanamori H, Jennings PC, Kisslinger C (eds) Invited contribution to The IASPEI International Handbook of Earthquake and Engineering Seismology. Academic Press, London, pp 1613-1614

Hansen PC, O'Leary DP (1993) The use of the L-curve in the regularization of discrete ill-posed problems. SIAM J Sci Comput 14:1487-1503. doi:10.1137/0914086 
Hirata N, Matsu'ura M (1987) Maximum-likelihood estimation of hypocenter with origin time eliminated using nonlinear inversion technique. Phys Earth Planet Int 47:50-61

Hukushima K, Nemoto K (1996) Exchange Monte Carlo method and application to spin glass simulation. J Phys Soc Jpn 65:1604-1608

Karato S, Jung H (1998) Water, partial melting and the origin of the seismic low velocity and high attenuation zone in the upper mantle. Earth Planet Sci Lett 157:193-207. doi:10.1016/S0012-821X(98)00034-X

Kinoshita S, Ohike M (2002) Scaling relations of earthquakes that occurred in the upper part of the Philippine Sea plate beneath the Kanto region, Japan, estimated by means of borehole recordings. Bull Seismol Soc Am 92:611-624. doi:10.1785/0120010134

Kodaira S, Sato T, Takahashi N, Ito A, Tamura Y, Tatsumi Y, Kaneda Y (2007a) Seismological evidence for variable growth of crust along the Izu intraoceanic arc. J Geophys Res 112:B05104. doi:10.1029/2006JB004593

Kodaira S, Sato T, Takahashi N, Miura S, Tamura Y, Tatsumi Y, Kaneda Y (2007b) New seismological constraints on growth of continental crust in the lzuBonin intraoceanic arc. Geology 35:1031-1034. doi:10.1130/G23901A.1

Obana K, Kamiya S, Kodaira S, Suetsugu D, Takahashi N, Takahashi T, Tamura Y (2010) Along-arc variation in seismic velocity structure related to variable growth of arc crust in northern Izu-Bonin intraoceanic arc. Geochem Geophys Geosyst 11:Q08012. doi:10.1029/2010GC003146

Obara K, Kasahara K, Hori S, Okada Y (2005) A densely distributed high-sensitivity seismograph network in Japan: Hi-net by National Research Institute for Earth Science and Disaster Prevention. Rev Sci Instrum 76:021301

Okada Y, Kasahara K, Hori S, Obara K, Sekiguchi S, Fujiwara H, Yamamoto A (2004) Recent progress of seismic observation networks in Japan -Hi-net, F-net, K-NET and KiK-net-. Earth Planets Space 56:xv-xxviii

Pozgay SH, Wiens DA, Conder JA, Shiobara H, Sugioka H (2009) Seismic attenuation tomography of the Mariana subduction system: implications for thermal structure, volatile distribution, and slow spreading dynamics. Geochem Geophys Geosyst 10:Q04X05. doi:10.1029/2008GC002313

Saito T, Sato H, Ohtake M (2002) Envelope broadening of spherically outgoing waves in three-dimensional random media having power law spectra. J Geophys Res. doi:10.1029/2001JB000264

Sato H (1989) Broadening of seismogram envelopes in the randomly inhomogeneous lithosphere based on the parabolic approximation: southeastern Honshu, Japan. J Geophys Res 94:17735-17747

Sato T, Kodaira S, Takahashi N, Tatsumi Y, Kaneda Y (2009) Amplitude modeling of the seismic reflectors in the crust-mantle transition layer beneath the volcanic front along the northern Izu-Bonin island arc. Geochem Geophys Geosyst 10:Q02X04. doi:10.1029/2008GC001990
Suyehiro K, Takahashi N, Ariie Y, Yokoi Y, Hino R, Shinohara M, Kanazawa T, Hirata N, Tokuyama H, Taira A (1996) Continental crust, crustal underplating and low-Q upper mantle beneath an oceanic island arc. Science 272:390-392

Takahashi T (2012) Three-dimensional attenuation structure of intrinsic absorption and wide-angle scattering of $S$ waves in northeastern Japan. Geophys J Int 189:1667-1680. doi:10.1111/j.1365-246X.2012.05438.x

Takahashi T, Sato H, Nishimura T (2008) Recursive formula for the peak delay time with travel distance in von Kármán type non-uniform random media on the basis of the Markov approximation. Geophys J Int 173:534545. doi:10.1111/j.1365-246X.2008.03739.x

Takahashi T, Obana K, Kodaira S, Suetsugu D, Takahashi N, Kamiya S, Tamura Y (2011) Random inhomogeneities in the northern Izu-Bonin arc estimated by tomographic inversion of peak delay times of S wave seismograms. J Geophys Res 116:B03303. doi:10.1029/2010JB007691

Takahashi T, Obana K, Yamamoto Y, Nakanishi A, Kodaira S, Kaneda Y (2013) The 3-D distribution of random velocity inhomogeneities in southwestern Japan and the western part of the Nankai subduction zone. J Geophys Res Solid Earth. doi:10.1002/jgrb.50200

Takahashi T, Obana K, Yamamoto Y, Nakanishi A, Kodaira S, Kaneda Y (2014) S-wave attenuation structure on the western side of the Nankai subduction zone: implications for fluid distribution and dynamics. J Geophys Res 119:7805-7822. doi:10.1002/2014JB011103

Tamura Y, Tatsumi Y, Zhao D, Kido Y, Shukuno H (2002) Hot fingers in the mantle wedge: new insights into magma genesis in subduction zones. Earth Planet Sci Lett 197:105-116

Tamura Y, Gill JB, Tollstrup D, Kawabata H, Shukuno H, Chang Q, Miyazaki T, Takahashi T, Hirahara Y, Kodaira S, Ishizuka O, Suzuki T, Kido Y, Fiske RS, Tatsumi Y (2009) Silicic magmas in the Izu-Bonin oceanic arc and implications for crustal evolution. J Petrol. doi:10.1093/petrology/egp017

Tatsumi Y, Shukuno H, Tani K, Takahashi N, Kodaira S, Kogiso T (2008) Structure and growth of the Izu-Bonin-Mariana arc crust: 2. Role of crust-mantle transformation and the transparent Moho in arc crust evolution. J Geophys Res 113:B02203. doi:10.1029/2007JB005121

Ueno H, Hatakeyama S, Aketagawa T, Funasaki J, Hamada N (2002) Improvement of hypocenter determination procedures in the Japan Meteorological Agency. Quart J Seismol 65:123-134 (in Japanese with English abstract)

Wessel P, Smith WHF (1998) New improved version of generic mapping tools released. EOS Trans AGU 79(47):579. doi:10.1029/98EO00426

Williamson IP (1972) Pulse broadening due to multiple scattering in the interstellar medium. Mon Not R Astron Soc 157:55-71

\section{Submit your manuscript to a SpringerOpen ${ }^{\odot}$ journal and benefit from:}

- Convenient online submission

- Rigorous peer review

- Immediate publication on acceptance

- Open access: articles freely available online

- High visibility within the field

- Retaining the copyright to your article

Submit your next manuscript at springeropen.com 